\title{
Tacrolimus-Induced Post-Transplant Diabetes Mellitus: A Case Report
}

\section{Jonathan Brend | Kevin Shotliff*}

*Correspondence: Kevin Shotliff

Address: Beta Cell Diabetes Centre, Chelsea and Westminster Hopsital, 369 Fulham Road, London

e-mail $\bowtie$ : Kevin.Shotliff@chelwest.nhs.uk

Received: 15 January 2021; Accepted: 29 January 2021

Copyright: (C) 2021 Brend J. This is an open-access article distributed under the terms of the Creative Commons Attribution License, which permits unrestricted use, distribution, and reproduction in any medium, provided that the original work is properly cited.

\section{ABSTRACT}

A 38-year-old man presents to hospital in diabetic ketoacidosis (DKA) after developing presumed post-transplant diabetes mellitus (PTDM) following an orthotopic heart transplant due to fulminant viral myocarditis. Amongst his immunosuppressant therapy he took tacrolimus, which is commonly used after solid organ transplantation. Whilst it is very effective in preventing rejection, it has a range of side effects including an increased risk of PTDM. Depending on the organ transplanted, PTDM can affect between 10 to $40 \%$ of solid organ transplants and patients taking tacrolimus have been shown to be more likely to develop this side effect (Chowdhury, 2019). During his admission with myocarditis, he also developed Covid-19, which has been anecdotally associated with the development of new-onset diabetes and increased presentations of DKA.

Keywords: Tacrolimus, Post-Transplant Diabetes Mellitus, Diabetic Ketoacidosis

\section{Clinical Presentation}

A 38-year-old man, with past medical history of asthma and Glucose-6 phosphate dehydrogenase deficiency (G6PD deficiency), received an orthotopic heart transplant as management for acute heart failure secondary to fulminant viral myocarditis treated with tacrolimus, mycophenolate mofetil and prednisolone as immunosuppression.

During that admission he also caught severe acute respiratory syndrome coronavirus 2 (SARSCoV-2). The patient was not known to be diabetic, however his father and mother both had a history of type 2 diabetes mellitus, both controlled with oral medication.

\section{Clinical Findings}

Two months post-transplant, he presented to the emergency department with lethargy and he was unable to give a coherent history. The paramedics reported that he began to develop nausea and vomiting during the previous day and had a one-week history of polyuria, polydipsia and $7 \mathrm{~kg}$ of weight 
loss with reduced appetite.

\section{Diagnostic Assessment}

On admission, he was found to have a severe metabolic acidosis with a venous gas $\mathrm{pH}$ of 7.12 , a bicarbonate of $6.5 \mathrm{mEq} / \mathrm{l}$, ketonaemic (blood ketones: $6.2 \mathrm{mmol} / \mathrm{L}$ ) and hyperglycaemic (blood glucose: $74.0 \mathrm{mmol} / \mathrm{L}$ ). He was also found to have an elevated Troponin I, but denied any cardiovascular symptoms. Furthermore, he was mildly hyperkalaemic with a potassium of $5.9 \mathrm{mmol} / \mathrm{L}$, with an eGFR of $17 \mathrm{ml} /$ minute.

An inpatient echocardiogram was performed which showed:

\begin{tabular}{|l|l|}
\hline Hyperdynamic LV systolic function (Normal LVEF 65-70\%) & Mild TR \\
\hline Mild to moderate concentric LVH & Normal LV cavity size \\
\hline Reduced RV longitudinal systolic function (TAPSE $1.4 \mathrm{~cm})$ & RV dilatation \\
\hline Preserved RV radial systolic function. & No obvious resting RWMAs \\
\hline Normal RA size by area $\left(17 \mathrm{~cm}^{2}\right)$ & Restrictive filling pattern \\
\hline IVC dilated $(2.5 \mathrm{~cm}$ ) with $>50 \%$ inspiratory collapse. & LA dilatation \\
\hline Estimated PASP $34.8 \mathrm{mmHg}+$ RAP (5-10 $\mathrm{mmHg})$ & No pericardial effusion \\
\hline
\end{tabular}

\section{Therapeutic Intervention}

The patient was admitted to the Intensive Care Unit, and started on a fixed rate intravenous insulin infusion, as well as IV fluids as per the hospital's DKA guidelines. On the day after his admission, his acidosis had resolved. After 24 hours he was stepped down to the Acute Assessment Unit, and started on a basal bolus insulin regimen, with Abasaglar and Novorapid, which was adjusted to the optimal doses with the help of the Diabetic Specialist Nurses.

\section{Follow-up and Outcomes}

The cardiac transplant team reviewed this patient, and the tacrolimus was stopped, but he remained on prednisolone. He will also be followed up in the endocrinology clinic, once face-to-face consultations resume.

\section{Discussion}

The most likely cause of the patient's symptoms are felt to be due to PTDM; induced by tacrolimus as part of his immunosuppressant therapy. Tacrolimus is a calcineurin inhibitor and is highly effective 
in preventing rejection. One of the side effects, however, is an increased likelihood of developing PTDM, with as many as up to 74\% of organ recipients effected (Biddle and Ahmed, 2019).

Tacrolimus works on cytotoxic T cells (Biddle and Ahmed, 2019), specifically through inhibition of the protein, phosphorylase calcineurin. Calcineurin works by dephosphorylating nuclear factor of activated T cells (NF-AT). This triggers NF-AT movement into T cell nuclei and the transcription of NFAT-dependant cytokines (Biddle and Ahmed, 2019; Chakkera et al., 2016), which stimulate T cell proliferation. Tacrolimus reduces $\mathrm{T}$ cell proliferation by binding to immunophilin FKBP-12 and the resulting complex prevents dephosphorylation by calcineurin of NF-AT (Biddle and Ahmed, 2019).

Tacrolimus and other calcineurin inhibitors are thought to raise the occurrence of PTDM through a variety of different mechanisms (Chakkera et al., 2016; Pereira et al., 2014). Calcineurin is expressed in pancreatic beta-cells and plays a key role in beta-cell growth and survival through expression of NFAT and insulin receptor substrate 2 (IRS2). IRS2 is also involved in insulin secretion via the P13K/Akt pathway. Therefore, calcineurin inhibitors induce diabetes through reduced beta-cell proliferation and increased apoptosis, as well as reduced insulin secretion (Chakkera et al., 2016).

Another mechanism of PTDM, is through calcineurin inhibitors' effect on adipocyte cells. Tacrolimus has been shown to reduce glucose uptake in human subcutaneous and omental adipocytes (Pereira et al., 2014). Furthermore, tacrolimus has been shown to reduce insulin sensitivity in peripheral tissues (Biddle and Ahmed, 2019). This can be explained by inhibition of the calcineurin/NFAT pathway, which has been hypothesised to promote transcription of insulin-resistant myosin fast fibres in skeletal muscle tissue (Chakkera and Mandarino, 2013).

One of the common side effects of tacrolimus is hypomagnesaemia. This electrolyte abnormality has been shown to be implicated in insulin resistance and hyperglycaemia and is therefore and independent risk factor for development of PTDM (Biddle and Ahmed, 2019).

In studies comparing the efficacy of tacrolimus and ciclosporin in preventing acute rejection and graft loss, tacrolimus has been superior (Ekberg et al., 2007). However, the majority of studies comparing the two report higher incidents of PTDM with tacrolimus (Chakkera and Mandarino, 2013).

A study suggests that pre-existing risk factors for developing diabetes mellitus also increase the risk of PTDM. Furthermore, there is evidence that higher doses of tacrolimus have been shown to carry an increased risk of PTDM (Biddle and Ahmed, 2019). Taking this into account, careful selection of both the most appropriate patients and dose are crucial when initiating tacrolimus therapy in order to lower this risk of PTDM. 
There is another potential cause for the patient's development of insulin-dependent diabetes. Recently there is growing anecdotal evidence that there may be a link between SARS-CoV-2 infection and the development of diabetes and increased presentations of DKA (Rubino et al., 2020).

Furthermore, in a study conducted in North West London, doctors compared the incidence of new diagnoses of type 1 diabetes (T1DM) in children during the first peak of the SARS-CoV-2 pandemic. They found a largely increased incidence of T1DM and higher rates of DKA compared to expected rates. Some of these children were found to either have current or previous infection with SARS-CoV-2 (Unsworth et al., 2020). It is proposed that as SARS-CoV-2 binds to angiotensin-converting enzyme 2 (ACE2) receptors (Hussain et al., 2020), which are found in pancreatic beta cells (amongst other organs), SARSCoV-2 may precipitate modified glucose metabolism that may lead to the onset of diabetes or increase the risk of developing diabetes through other means. Moreover, other coronaviruses that bind to ACE2 receptors have been associated with ketosis-prone diabetes previously (Yang et al., 2010). Currently, there is no proven direct link between SARS-CoV-2 and new onset T1DM and we do not yet know whether these new cases of diabetes are temporary or permanent. It is also unclear whether this is a new type of diabetes, as opposed to traditional type 1 or type 2 diabetes, and whether only active SARSCoV-2 infection increases the risk of developing diabetes, or if any increased risk persists post-infection. To answer all of these questions, a global database of patients with new-onset diabetes associated with SARS-CoV-2 (known as the CoviDIAB Project) has been created (Rubino et al., 2020).

\section{Conclusion}

This case showing potential iatrogenic harm from a commonly used immunosuppressant, with a calcineurin inhibitor potentially influencing several individual steps in order to induce PTDM. Whilst it is important to give effective immunosuppression in transplant patients, it is also vital to consider the potential adverse effects of such treatment, both the agent used and the dose given. Patients may need to be counselled about the risks of developing PTDM, as well as how to recognise the symptoms of diabetes; especially in those with pre-existing risk factors.

Acknowledgements: The authors received no financial compensation for this case report and have no conflicts of interest.

\section{References}

Biddle K and Ahmed S. Tacrolimus. Practical Diabetes 2019; 36: 33-35.

Chakkera $\mathrm{H}$ and Mandarino L. Calcineurin Inhibition and New-Onset Diabetes Mellitus After Transplantation. Transplantation Journal 2013; 95: 647-652. 
Chakkera H, Kudva Y, Kaplan B. Calcineurin Inhibitors: Pharmacologic Mechanisms Impacting Both Insulin Resistance and Insulin Secretion Leading to Glucose Dysregulation and Diabetes Mellitus. Clin Pharmacol Ther 2016; 101: 114-120.

Chowdhury T. Post-transplant diabetes mellitus. Clinical Medicine 2019; 19: 392-395.

Ekberg H, Tedesco-Silva H, Demirbas A, Vítko Š, Nashan B, Gürkan A, Margreiter R, Hugo C, Grinyó JM, Frei U, Vanrenterghem Y. Reduced Exposure to Calcineurin Inhibitors in Renal Transplantation. N Engl J Med 2007; $357: 2562-2575$.

Hussain M, Jabeen N, Raza F, Shabbir S, Baig AA, Amanullah A, Aziz 0042. Structural variations in human ACE2 may influence its binding with SARS-CoV-2 spike protein. J Med Virol 2020; 92: 1580-1586.

Pereira MJ, Palming J, Rizell M, Aureliano M, Carvalho E, Svensson MK, Eriksson JW. Cyclosporine A and Tacrolimus Reduce the Amount of GLUT4 at the Cell Surface in Human Adipocytes: Increased Endocytosis as a Potential Mechanism for the Diabetogenic Effects of Immunosuppressive Agents. J Clin Endocrinol Metab 2014; 99: E1885-E1894.

Rubino F, Amiel SA, Zimmet P, Alberti G, Bornstein S, Eckel RH, Mingrone G, Boehm B, Cooper ME, Chai Z, Del Prato S. New-Onset Diabetes in Covid-19. N Engl J Med 2020; 383: 789-790.

Unsworth R, Wallace S, Oliver NS, Yeung S, Kshirsagar A, Naidu H, Kwong RM, Kumar P, Logan KM. New-Onset Type 1 Diabetes in Children During COVID-19: Multicenter Regional Findings in the U.K. Diabetes Care 2020; 43: e170-171.

Yang JK, Lin SS, Ji XJ, Guo LM. Binding of SARS coronavirus to its receptor damages islets and causes acute diabetes. Acta Diabetol 2010; 47: 193-199. 